\title{
The support of bounded saddles
}

\author{
A. R. MacGregor, PhD, FDS, FDS, DRDRCS(Edin) \\ T. P. G. Miller, \\ University of Glasgow Dental School \\ J. W. Farah, Phd, DDS \\ University of Michigan School of Dentistry, USA
}

\begin{abstract}
This article reviews the literature on planning the support of bounded saddles of removable partial dentures or fixed bridges. Three-dimensional photoelastic analysis was used to examine stresses on removable partial dentures of different designs, and possible model structure changes raised for further investigation.
\end{abstract}

\section{INTRODUCTION}

When planning the support of bounded saddles of removable partial dentures or fixed bridges there is a lack of reliable scientific data on which accurate decisions can be based. Many of the designs of fixed and removable appliances have evolved over the years from a mix ture of engineering theory, clinical experience and empirical judgement. This investigation has been carried out to examine some of the variables and is a continuation of work previously reported by MacGregor et al. $(1978,1980)$ who examined stresses on removable partial dentures of different designs by means of three-dimensional photoelastic analysis.

\section{REVIEW OF THE LITERATURE}

The problems of the support deficit of bounded saddles were discussed by Watt et al. (1958) who investigated various factors involved in the design of saddle support.

Few experiments have been performed to compare the merits of removable and fixed partial dentures in situations with bounded saddles (Nagasawa and Tsuru, 1973), and it is generally agreed that when selecting abutments for fixed or removable prostheses few definite rules can be applied (Tylman and Malone, 1978). The latter-mentioned authors arbitrarily designated spans (saddles) as short (one pontic), medium (two pontics) and long (several pontics) and stated that two abutment teeth can support two pontics. Thus the combined periodontal area of the abutment teeth should be equal to or greater than the periodontal area of the tooth or teeth to be replaced.

In a statement designated by Johnston et al. (1971) as Ante's Law, the requirement was proposed that the root surface area of the abutment teeth should equal or surpass that of the teeth being replaced with pontics (Ante, 1926). According to Kantorowicz (1979), this is good clinical advice and a convenient way of assessing the number of abutments is to count how many tecth are to be replaced and add one to the total. The area of root needed for support, however, will depend on the length of the span to be supported and the amount of leverage to fall on the span. Roberts (1980) is of the opinion that the longer the span the 
greater the load which will be placed on the abutment teeth and obviously there comes a stage where a removable prosthesis is indicated so as to obtain some degree of tissue support and thus avoid overloading the abutments. However, this poses the problem of sharing load between compressible tissue (mucosa) and tissue with very limited yield (periodontium). Methods devised to solve this problem are often less than satisfactory.

In addition to the increased load placed on the periodontium by a long-span saddle or bridge, longer spans are less rigid. Beams flex to some extent, the deflection varying directly with the cube of the length and inversely with the cube of the occlusogingival height of the pontic (Shillingburg et al., 1976).

According to McLean (1980), as the length of a beam changes, the transverse deflection increases as the cube of the length. Doubling the length of a span will allow eight times as much deflection for a given force. For example, if a one-tooth pontic bridge is compared with a two-tooth pontic bridge, and the design of the pontics and connectors remain unchanged, then the two-tooth bridge will bend eight times as much as the one-tooth bridge.

The above statements are true for beams of homogenous material and uniform crosssections. Where the beam is long in proportion to its height, the length/height ratio being eight or more and the width approximately the same order as the depth, then the deflection is calculated from the equation:

$$
\text { deflection }=\frac{F L^{3}}{C E I}
$$

$F$ is the force applied

$L$ is the length

$C$ is a constant dependent on end conditions

$E$ is the modulus of elasticity

$I$ is the moment of inertia about the centroidal axis

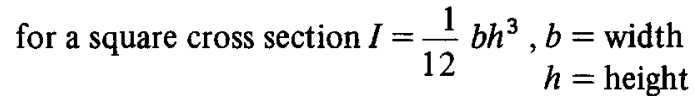

The physical properties and behaviour of the tissues which surround abutment teeth are important when investigating methods of supporting bounded saddles of bridges or removable partial dentures. These matters have been the subject of various investigations in recent years (Parfitt, 1967; Johnson, 1977; Wills, 1977; Picton and Wills, 1978).

Friedman (1973) emphasized the importance of directing loads axially rather than either horizontally or torsionally in bounded-saddle situations. During axial loading, the vertical stress is resisted by the oblique fibres which run diagonally from cementum coronally into alveolar bone and thus function as a suspensory sling. While vertical occlusal forces are transmitted as pressure through the root they are converted by the stretching of the oblique fibres into tensile stresses. Thus occlusal pressure is dissipated favourably as tension. On the other hand, with transverse occlusal forces, the axis of rotation stretches half the oblique fibres while the other half are compressed.

If the direction of the load line lies within the lateral border of the root, stress is directed vertically on the periodontium; if the load is directed outside the root, then lateral or tipping stresses are induced. The type of appliance may have a bearing on this and Lundgren et al. (1975), reporting a functional analysis of results in a group of 18 patients with reduced 
periodontal support, concluded that fixed bridges are superior to removable partial dentures.

When evaluating abutments the root configuration, in addition to periodontal area and periodontal health, is important. From a mechanical point of view either a round crosssection or a regular taper may be considered unfavourable.

Different methods may be used to examine the effects and results of loads on bounded saddles. Some of these are done directly in the mouth while others are managed under laboratory conditions.

The area of applied science covered by the term stress analysis aims to determine the distributions of stress or deflections in any structure when loads are applied. Biological parts and structures, when loaded, should also be able to be analysed using the same general principles as inert objects. However, in living tissues there are different and usually complex conditions and properties of materials. It is for this reason that the theoretical modelling of a clinical situation is often the most difficult and controversial area in stress analysis (Sutcliffe and Atherton, 1980). By nature of the technique, the laboratory modelling is simplified but, of course, is then liable to be incomplete although not necessarily inaccurate or unreliable. Nevertheless, a vital tooth in a mandible is not exactly the same as a metal bolt in a block of wood, although they may well behave in a similar way under certain circumstances.

Kratochvil and Caputo (1974) used a photoelastic replica to test the distribution of stress on a removable partial denture but one of the saddles was a free-end (distal extension) and this may have influenced the results on the other saddle which was bounded.

Sutherland et al. (1980), in a two-dimensional photoelastic stress analysis of bounded saddles, found that stress distribution was more uniform when $(a)$ the sites of load application were increased in number and $(b)$ the load was positioned nearer the abutment with the greater root surface area.

Graf et al. (1974) used a piezo-electric transducer incorporated in the crown of a molar after endodontic treatment to measure masticatory forces on the tooth during chewing.

Hewitt (1981), in pointing out that small forces applied to teeth generate stress not only adjacent to the source but also at sites remote from the point of application, suggested that holographic techniques may be used to study the stress patterns. This was previously pointed out by Young and Altschuler in 1977.

Other investigators who examined the effects of loads on partial dentures and abutment teeth include Christidou et al. (1973), Goodkind (1973), Cecconi (1974), Maxfield et al. (1979) and McCartney (1980). Each of these reports, however, was restricted to dentures with free-end saddles. Craig and Farah (1978) also examined dentures with free-end saddles but they used a method involving two-dimensional finite element analysis.

\section{METHOD}

The present study comprised a photoelastic analysis of epoxy-resin models which carried removable saddles supported on abutment teeth in a number of different ways.

The investigation was restricted to bounded saddles. The object was to confirm whether the technique was sufficiently sensitive and accurate for valid conclusions to be made with respect to different tooth-supported situations and different loading positions.

A master cast was made in the laboratory, the missing mandibular teeth being both first molars and both third molars; thus the bounded saddles were one-tooth spaces and the main abutment teeth were the second premolars and second molars. A silicone mould was made 


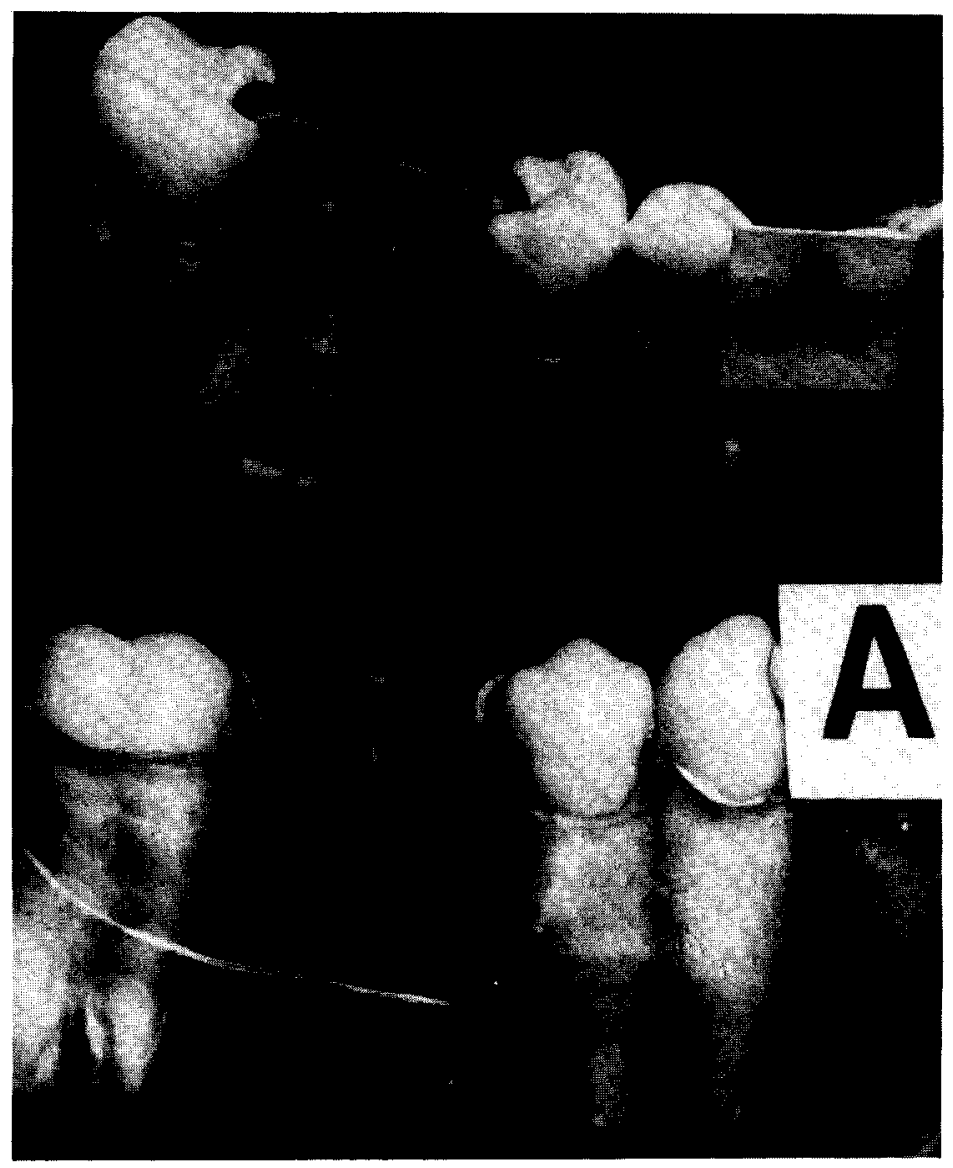

Fig. 1. Mandibular test bridge (design A) showing reinforced pontic.

from the cast and into the mould were placed typodont teeth made of urea-formaldehyde polymer. The roots were coated with an even thickness of silicone rubber to represent a pseudoperiodontal membrane.

Epoxy-resin models were made from this mould, the resin consisting of Epon 828 and an amine hardener Jeffamine D400 in the ratio of 2 to 1 poured under vacuum. The resin was allowed to cure at room temperature for three days.

Two test bridges of six different designs were made in cobalt chromium alloy (Wisil M) by following accepted laboratory procedures. The test bridges were used only when the fit was judged to be clinically acceptable. The pontics were reinforced with vertical struts (Fig. 1) to be as rigid as possible. An indentation on the occlusal surface was made to receive the end of the loading rod. None of the bridges contacted the saddle area between the abutment teeth.

\section{The design of the test bridges}

The different designs were as follows:

A, the small occlusal rests occupied the mesial and distal fossae of the two abutments immediately adjacent to the pontic (Fig. 1). 


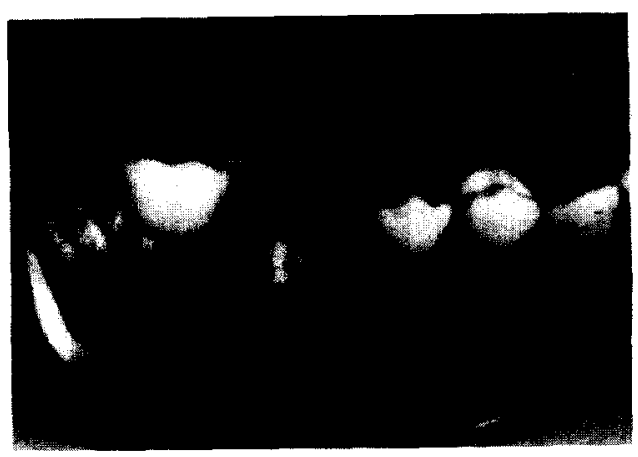

Fig. 2. Design B: rests extended as overlays.

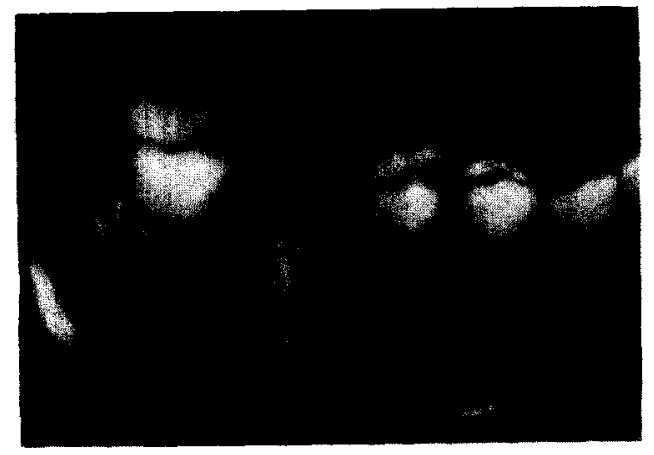

Fig. 3. Design C: occlusal rests on mesial fossae of abutments.

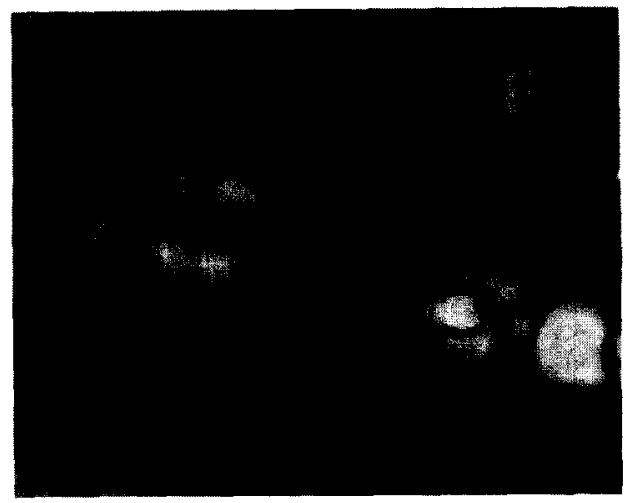

Fig. 5. Design E: assessed the effect of clasps on distribution of load.
Fig. 4. Design D: occlusal rests extended to cover a larger area than $\mathrm{A}$ or $\mathrm{C}$.

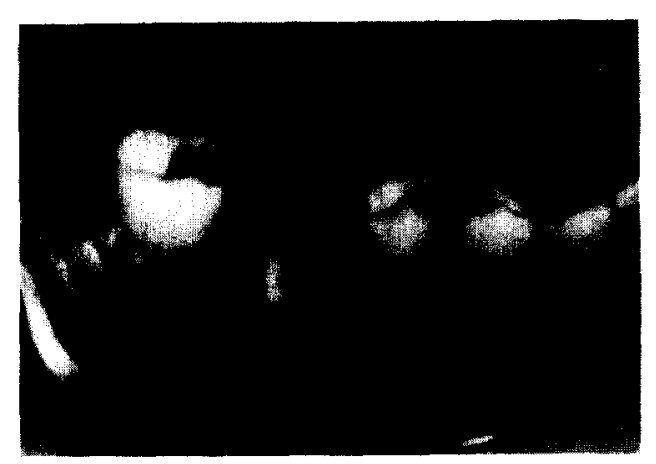

B, the rests extended over the occlusal surfaces as overlays (Fig. 2).

$\mathrm{C}$, the rests occupied the mesial fossae of the molar and premolar abutments. There was no contact against distal premolar (Fig. 3).

D, the molar rest extended to the central fossae while the anterior rest lay on two premolar abutments. There was no contact on distal premolar (Fig. 4).

$\mathrm{E}$, this bridge incorporated occlusally approaching clasps on both abutment teeth. The rests occupied the distal fossae of the premolar, and the mesial and distal fossae of the molar (Fig. 5).

F, short occlusal rests occupied distal premolar and mesial molar, with gingivally approaching buccal and lingual clasps (Fig. 6).

Each photoelastic model was placed in a loading jig in a stress-freezing oven. A load of $2.5 \mathrm{~kg}$ was then applied in the centre of each bridge (Fig. 7) and the stresses frozen in the model at $50^{\circ}$. Each model was then examined and the fringes calculated at various predetermined points. 


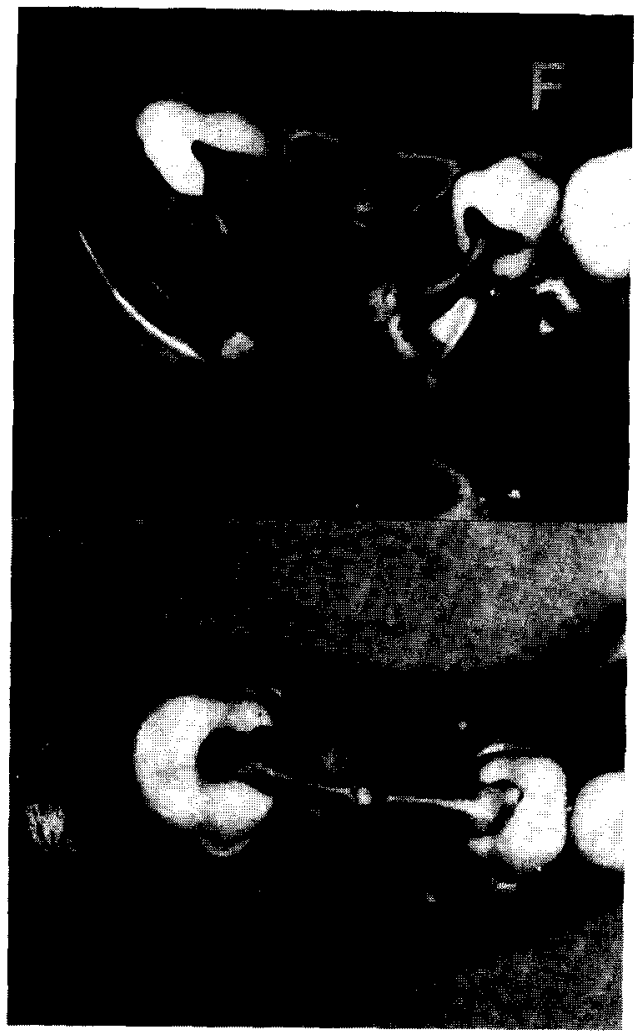

Fig. 6. Design F: incorporated gingivally approaching clasps which were made thick to provide bracing.

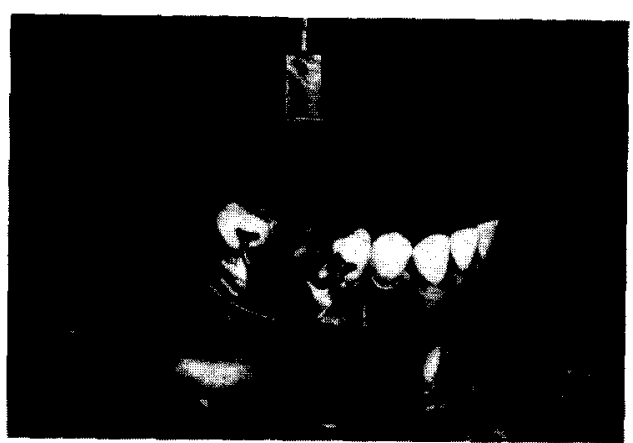

Fig. 7. A load of $2.5 \mathrm{~kg}$ being applied to one of the test bridges in the stress-freezing oven.

\section{RESULTS}

Fig. 8 is an example of the fringes observed at the apex of the premolars and second molar by loading one of the test bridges. In this illustration, the maximum fringe order at the apex of the first premolar was $N=4.0$ while at the apex of the second premolar $N=7$. The fringe order at the molar mesial root apex was $N=8.5$ and $N=6.3$ at the distal root apex.

Fig. 9 depicts the maximum fringe order determined at each of the points denoted on the horizontal axis. Each result is an average of four readings. The fringe order at the apex of the second premolar with the short rests (design A) was $N=14$ while that with full coverage (design B) $N=10 \cdot 3$.

At the apex of the mesial root of the second molar, $N=11$ for model A and $N=8$ for model B. At the apex of the distal root, $N=10$ for model A and $N=8.2$ for model B. Overall, the fringe order was higher for model A with short rests than for model B with the full coverage overlay, the latter showing a more even distribution of stress.

The maximum fringe orders for models $\mathrm{C}$ and $\mathrm{D}$ are drawn in Fig. 10. These values are averages from four readings. In general, higher fringe orders were obtained for model $\mathrm{C}$ than for model D. For example, at the apex of the second premolar the fringe order was $N=10$ for 

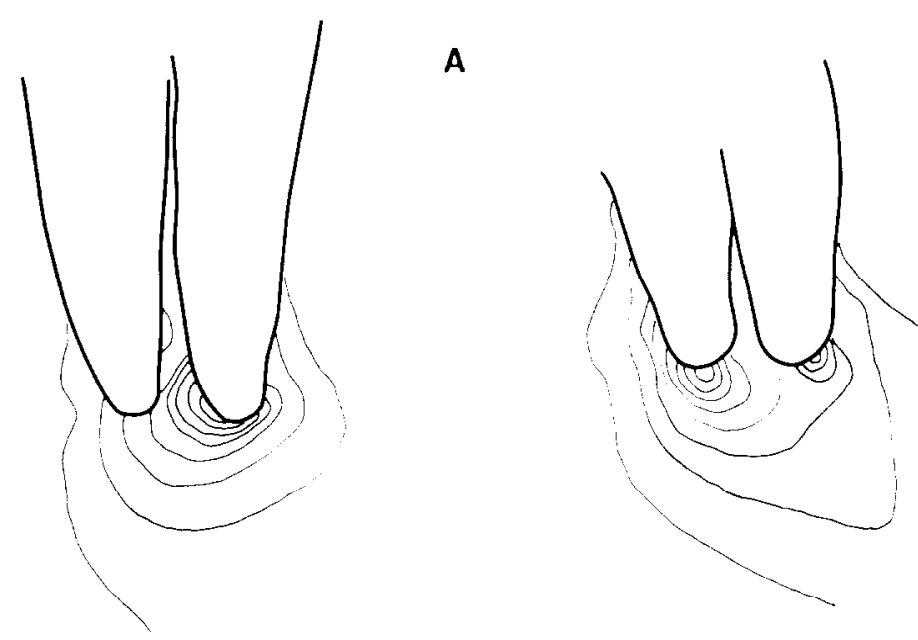

Fig. 8. A diagram of the fringes around the abutment teeth resulting from loading of test bridge design $A$.

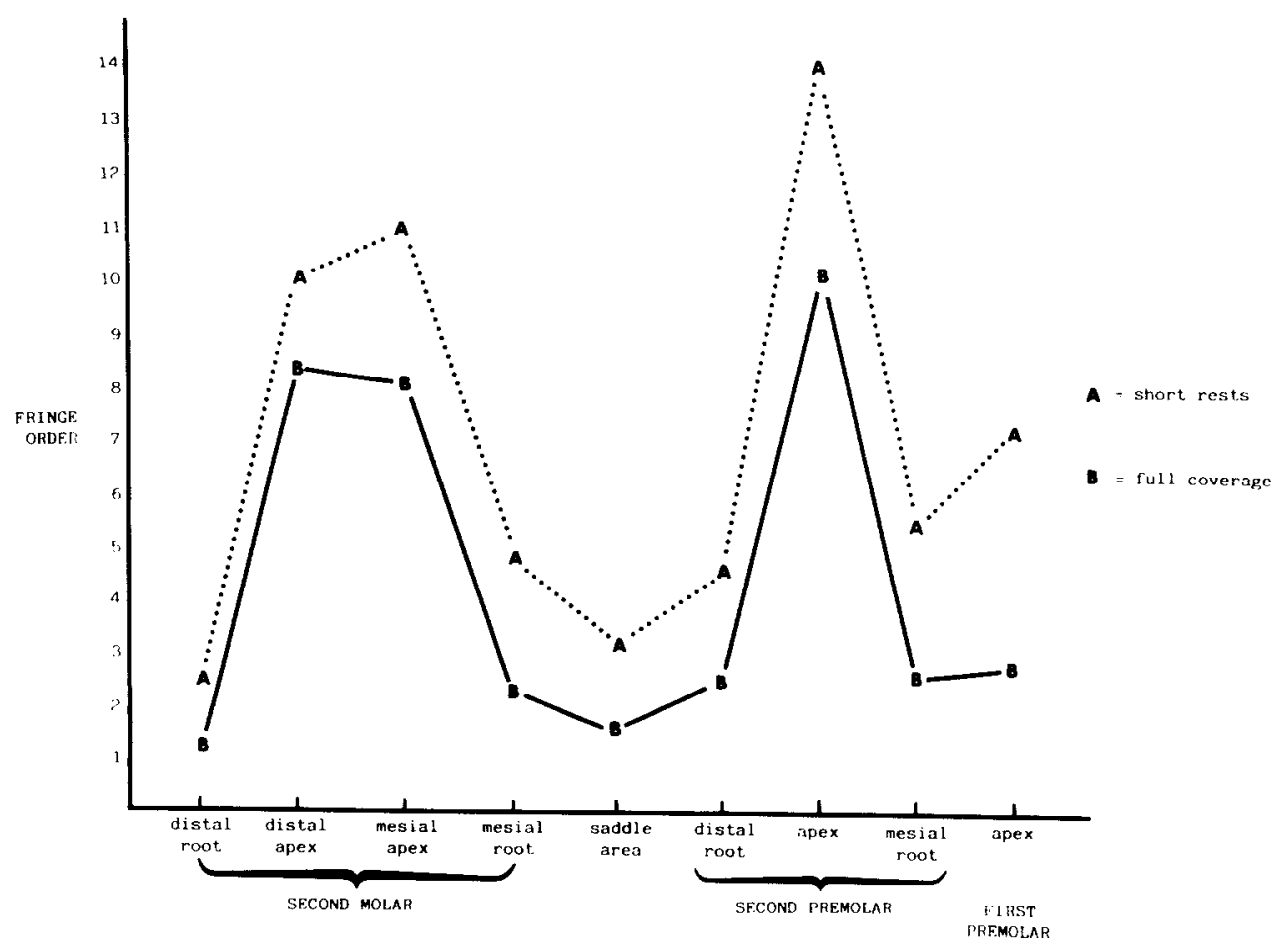

Fig. 9. Comparison of fringe orders in models with designs $A$ and $B$. The dotted lines connect the results of design $A$ at various tooth positions; the solid line denotes the fringe orders of design $B$ which show less peaking and a more even distribution of stress. Note that fringe orders could be determined in the saddle area although there was no contact between the pontic and the surface of the model. 


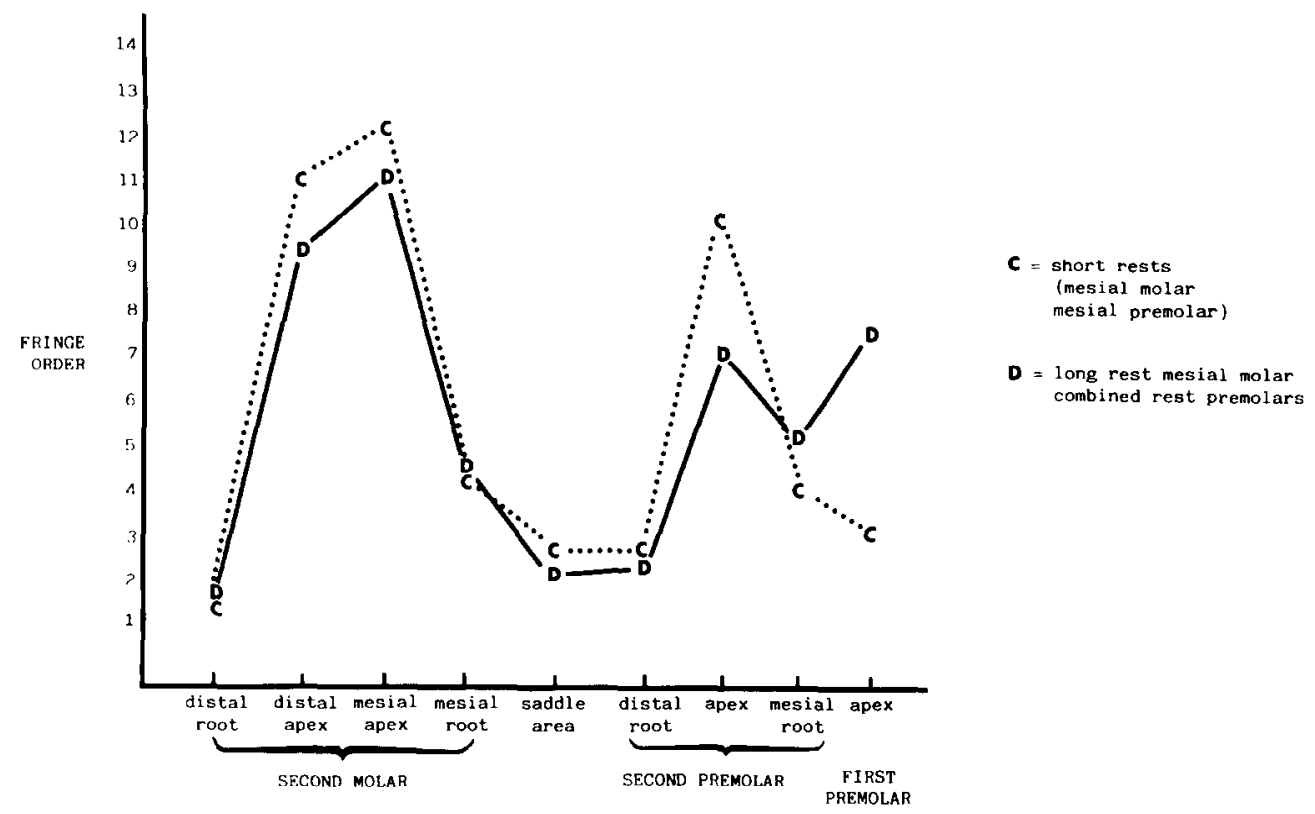

Fig. 10. Comparison of fringe orders in models with designs $C$ and $D$. The dotted lines connect the results of design $C$ at various tooth positions. The fringe orders for $D$ show a more even stress distribution, particularly at the premolar apices.

model $\mathrm{C}$ and $N=7$ for model $\mathrm{D}$. The fringe order at the apex of the first premolar was higher in model $\mathrm{D}, N=7 \cdot 5$, than model $\mathrm{C}$ where $N=3$. This was mainly due to the fact that design $D$ had a double rest on the mesial of the second premolar and the distal of the first premolar. The differences in the fringe order at the apices of the second molar were less pronounced, probably because the extension of the rest in design $\mathrm{D}$ was the only difference in design.

The differences in fringe orders between models $\mathrm{E}$ and $\mathrm{F}$ were not as clear. These two designs were essentially similar except for the clasping arrangement which was mainly occlusal as in Fig. 5, and gingival as in Fig. 6. The design in models $\mathbf{E}$ also had a distal molar rest but this did not seem to affect the stress distribution to any great extent although the fringe order at the distal root of the second molar was increased by an average of $N=0.5$. Originally, the difference in clasping was devised with the possibility of applying oblique loads from the buccal or lingual direction but in this particular test the loads were applied vertically; the bracing effect of clasps would be ineffective in this directional load.

\section{DISCUSSION}

In any photoelastic investigation the accuracy of model preparation is extremely important when one wishes to compare one model with another. Great care was taken to ensure the proper positioning of the teeth, and the uniformity in thickness of the pseudoperiodontal membrane. Thus, photoelastic model preparation tends to be time-consuming. However, it is true that if care is applied in preparation of the models the results will be accurate and 


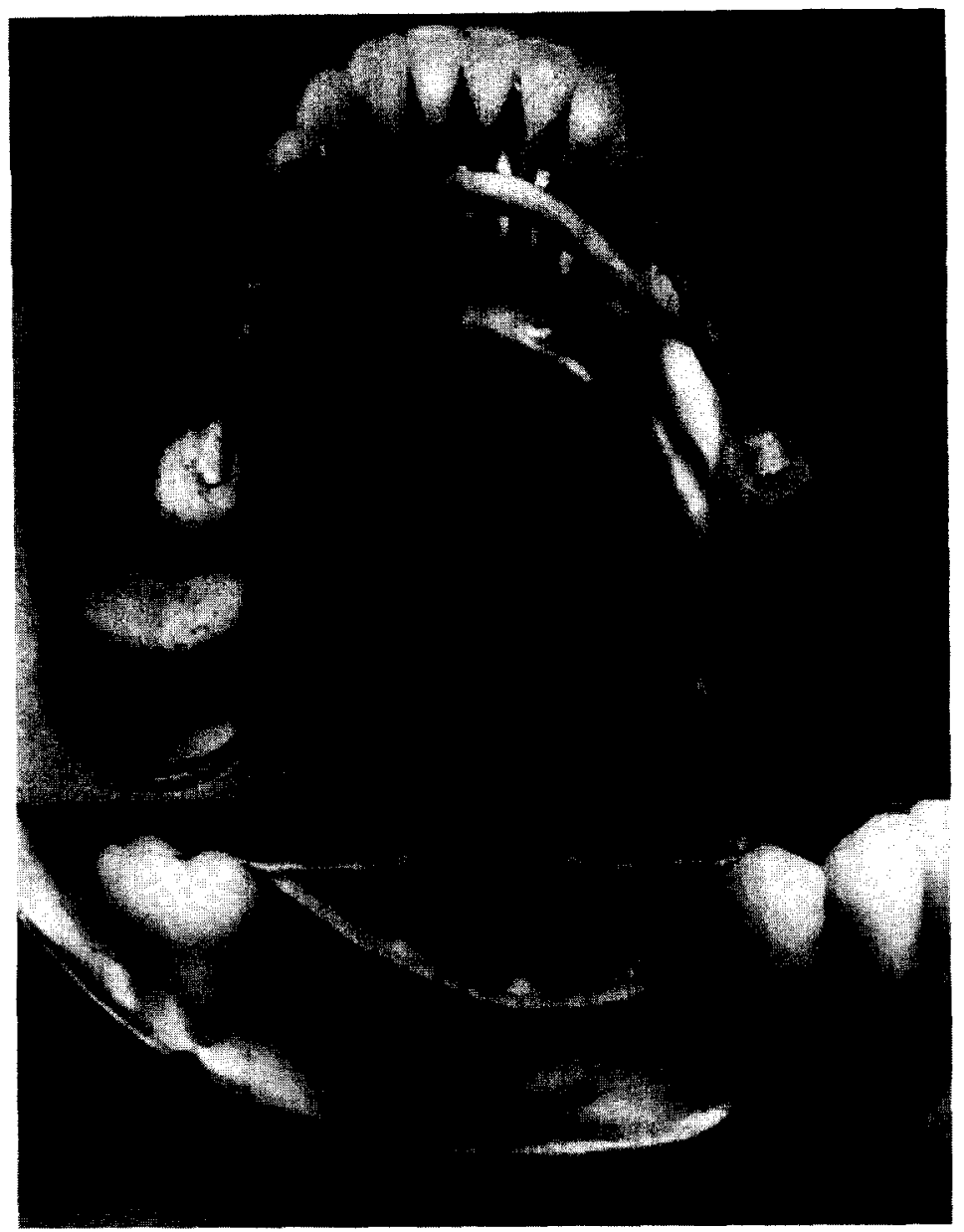

Fig. 11. Long bounded saddle. Photoelastic model and test bridge with long bounded saddles connected by a lingual bar. Three teeth are missing on each side. Load is applied in the middle of the saddle, which is supported on first premolar and third molar.

reproducible. Although three-dimensional photoelasticity is a versatile method for stress analysis, it is not very sensitive. For example, in this study, when occlusally approaching clasps were compared to gingivally approaching clasps practically no difference in the stress distribution was observed. Thus, subtle differences in the design of a partial denture will probably not be detected in a three-dimensional photoelastic model. Differences in the stresses were observed when a bridge with short rests was compared to a bridge with full coverage as shown in Fig. 9. The fringe order at the apex of the second premolar was about 27 per cent higher for the short rests when compared to full coverage. Similarly, at the apex of the second molar the fringe order at the inesial root apex was 28 per cent higher and 18 per cent higher at the distal root. One can observe from Fig. 9 that the fringe order was higher at each point that was selected. A possible explanation for the higher stress in the 


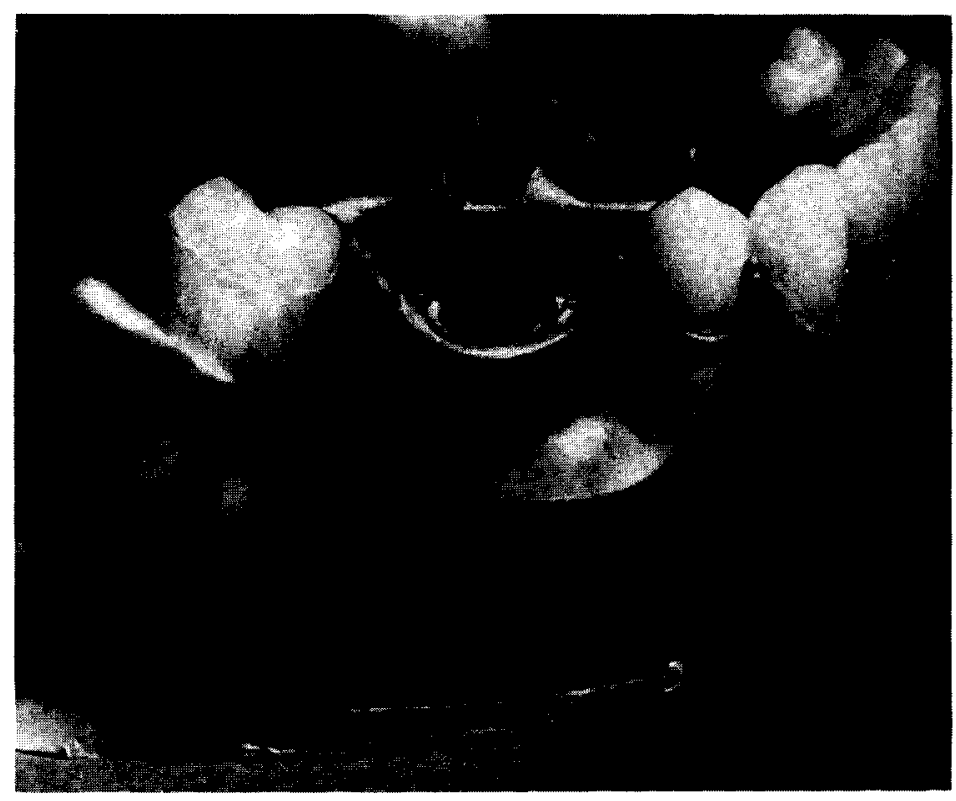

Fig. 12. Short bounded saddle. Model with bridges to test stress distribution from bounded saddles where first molars and second premolars are missing. The saddles are connected by a lingual bar.

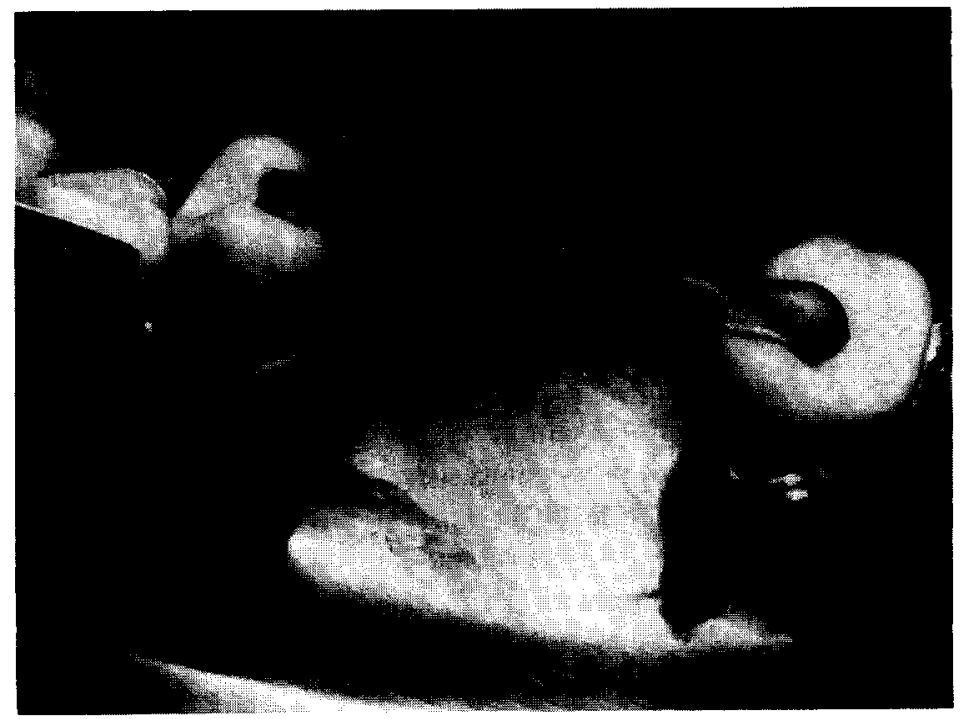

Fig. 13. Design of test bridge used to examine the load distribution by changing the position of the point of application of load. 
model where the rests are short is the existence of a tilting moment on each of the abutment teeth. The stress is the result of not only the axial load but also a tilting or bending load. The design with the full coverage, on the other hand, seems to transfer most of the load axially and very little, if any, tilting moment results. This would explain why the stresses are higher for model A with the short rests as compared to model B with full coverage.

The bridge designs for models $\mathrm{C}$ and $\mathrm{D}$ were similar with the exception that $\mathrm{D}$ had a double rest on the distal of the first premolar and mesial of the second premolar. The double rest tended to distribute the stress over an additional abutment and thus slightly lower the stress on the other abutments as seen in Fig. 10. The stress at the apex of the second premolar was about 30 per cent higher for model $\mathrm{C}$. Since design $\mathrm{C}$ had minimal contact with the first premolar when compared to design $\mathrm{D}$, the stress at the apex of the first premolar was about 60 per cent higher. The differences in stress at the apices of the second molar for both designs were not as dramatic because of the small difference in design of the molar occlusal rest.

\section{Recent investigations}

Two questions appear to be obvious as a result of the work reported:

1. What is the effect of increasing the length of the tooth-supported beam?

2. What is the effect of changing the point of application of the load?

These matters are now being examined. Test bridges of different length have been produced and are shown in Figs. 11 and 12 .

Models with saddles where two teeth are missing are being used to examine the effect of changing the point of load application. The bridges are produced with recesses to receive the loading jig at different points (Fig. 13).

\section{Acknowledgements}

Our thanks are due to Mr John B. Davies and the staff of the Department of Dental Photography and Illustration, University of Glasgow Dental School.

\section{REFERENCES}

Ante I. H. (1926) The fundamental principles of abutments. Mich. State Dent. Soc. Bull. 8, 14.

Cecconi B. T. (1974) Effect of rest design on transmission of forces to abutment teeth. $J$. Prosthet. Dent. 32, 141.

Christidou L., Osborne J. and Chamberlain J. B. (1973) The effects of partial denture design on the mobility of abutment teeth. Br. Dent. J. 135, 9.

Craig R. G. and Farah J. W. (1978) Stresses from distal-extension removable partial dentures. J. Prosthet. Dent. 39, 274.

Friedman J. (1973) Case analysis in the solution of bounded space problems. J. Prosthet. Dent. 30, 668 .

Goodkind R. J. (1973) The effects of removable partial dentures on abutment tooth mobility. J. Prosthet. Dent. 30, 139.

Graf H., Grassl H. and Aeberhard H. J. (1974) A method of measurement of occlusal forces in three directions. Helv. Odontol. Acto 18, 7.

Hewitt A. B. (1981) Holographic investigation of bone response to small forces. $B r . J$. Orthodont. 8, 43. 
Johnson G. H. (1977) Biomechanical Properties of the Human Periodontal Ligament. MS thesis, University of Washington.

Johnston J. F., Phillips R. W. and Dykema R. W. (1971) Modern Practice in Crown and Bridge Prosthodontics, 3rd ed. Philadelphia, Saunders, p. 11.

Kantorowicz G. F. (1979) Inlays, Crowns and Bridges, 3rd ed. Bristol, Wright, p. 140.

Kratochvil F. J. and Caputo A. A. (1974) Photoelastic analysis of pressure on teeth and bone supporting removable partial dentures. J. Prosthet. Dent. 32, 52.

Lundgren D., Nyman S., Heijl L. et al. (1975) Functional analysis of fixed bridges on abutment teeth with reduced periodontal support. J. Oral Rehabil. 2, 105.

McCartney J. W. (1980) Motion vector analysis of an abutment for a distal extension removable partial denture. J. Prosthet. Dent. 43, 15.

McLean J. W. (1980) The Science and Art of Dental Ceramics. Chicago, Quintessence, vol. 2, p. 332.

MacGregor A. R., Miller T. P. G. and Farah J. W. (1978) Stress analysis of partial dentures. J. Dent. 6, 125 .

MacGregor A. R., Miller T. P. G. and Farah J. W. (1980) Stress analysis of mandibular partial dentures with bounded and free-end saddles. J. Dent. 8, 27.

Maxfield J. B., Nicholls J. I. and Smith D. E. (1979) The measurement of forces transmitted to abutment teeth of removable partial dentures. J. Prosthet. Dent. 41, 134.

Nagasawa T. and Tsuru H. (1973) A comparative evaluation of masticatory efficiency of fixed and removable restorations replacing first molars. J. Prosthet. Dent. 30, 263.

Parfitt G. J. (1967) The Mechanisms of Tooth Support. Bristol, Wright, pp. 154-156.

Picton D. C. A. and Wills D. J. (1978) Viscoelastic properties of the periodontal ligament and mucous membrane. J. Prosthet. Dent. 40, 263.

Roberts D. H. (1980) Fixed Bridge Prostheses, 2nd ed. Bristol, Wright, p. 39.

Shillingburg H. T., Hobo S. and Whitsett L. D. (1976) Fundamentals of Fixed Prosthodontics. Chicago, Quintessence, p. 21.

Sutcliffe W. J. and Atherton J. D. (1980) The mechanics of tooth mobility. Br. J. Orthodont. 7,171 .

Sutherland J. K., Holland G. A., Sluder T. B. et al. (1980) A photoelastic analysis of the stress distribution in bone supporting fixed partial dentures of rigid and non-rigid designs. J. Prosthet. Dent. 44, 616.

Tylman S. D. and Malone W. F. P. (1978) Theory and Practice of Fixed Prosthodontics, 7th ed. St Louis, Mosby.

Watt D. M., MacGregor A. R., Geddes M. et al. (1958) A preliminary investigation of the support of partial dentures and its relationship to vertical loads. Dent. Practit. 9, 2.

Wills D. J. (1977) A Study of Some Mechanical Properties of Tooth Supporting Tissues in Monkeys (Macacus irus). PhD thesis, University of London.

Young J. M. and Altschuler B. R. (1977) Laser holograpy in dentistry. J. Prosthet. Dent. 38, 216. 